\title{
Methicillin Resistant Staphylococcus aureus Nasal Carriage among Health Care Workers at Rawalpindi Institute of Cardiology, Rawalpindi, Pakistan
}

\author{
Arif Maqsood Ali ${ }^{*}$, Azhar Mehmood Kiyani ${ }^{2}$, Agha Babar Hussain ${ }^{2}$ \\ ${ }^{1}$ Department of Pathology and Blood Bank, Rawalpindi Institute of Cardiology, Pakistan \\ ${ }^{2}$ Department of Medicine, Rawalpindi Institute of Cardiology, Pakistan
}

\begin{abstract}
Background

Staphylococcus aureus (S. aureus) is a virulent pathogen that is currently the most common cause of infections in hospitalized patients. Health care workers (HCWs) play a significant role in the epidemiology of Methicillin Resistant S. aureus (MRSA) infection. HCWs act as a vector for transmission of MRSA as they work at the interface between hospital and the community. Transmission of MRSA in health care environment is usually by contact between patients through hands, cloths or equipment of HCWs.
\end{abstract}

Objectives

To determine the percentage of HCW's having MRSA carriage at a tertiary care hospital in Rawalpindi Pakistan.

Materials and methods

Nasal swabs from anterior nares of HCWs were cultured on Blood agar and Macconkey Agar and Mannitol Salt Agar (MSA) and incubated at $35^{\circ} \mathrm{C}$ for $24-48$ hours. S. aureus growth was confirmed by colony morphology, gram staining and biochemical tests. MRSA were identified by observing zone of inhibition around Cefoxitin disc on Mueller Hinton Agar (MHA) according to CLSI guidelines.

Results

The frequency of nasal carriage of MRSA was $6.5 \%$. High nasal carriage was seen in ITC among the nursing staff.

Conclusion

High frequency of MRSA nasal carriage in ITC staff reflects poor hand hygiene and urgent need for infection control measures.

Keywords:

Health care workers $\bullet$ Methicillin resistance $\bullet$ Staphylococcus aureus $\bullet$ Nasal carriage

\section{Introduction}

S. aureus is a major cause of community and healthcare infections and is one among leading etiologic agents for skin and soft tissue infections, food poisoning, endocarditis, osteomyelitis and life-threatening postsurgical infections [1]. Staphylococcus aureus is both a human skin and mucosae commensal but also a frequent cause of serious infections with high morbidity, mortality, and healthcare-associated costs [2].
Treatment of infection caused methicillin resistant $\mathrm{S}$. aureus has become more problematic and challenging, as it is resistant to all $\beta$-lactam antibiotics thereby significantly limiting the treatment options. With a few exceptions, the incidence of nosocomial infection caused by MRSA continues to increase worldwide [3].

Infections caused by MRSA strains are associated with longer hospital stay, prolonged antibiotic administration, poor hand hygiene, unresponsive behaviour of hospital staff towards patients, presence of MRSA among patients and contaminated environmental surfaces. Colonized HCWs can act

Correspondence to: Arif Maqsood Ali, Department of Pathology and Blood Bank, Rawalpindi Institute of Cardiology, Rawalpindi, Punjab, Pakistan, Tel: +923217415605, +92519281111; E-mail: drarifmaqsoodali@hotmail.com

Received: January 16, 2020; Accepted: June 22, 2020; Published: June 29, 2020

Copyright: ( 2020 Ali AM. This is an open-access article distributed under the terms of the Creative Commons Attribution License, which permits unrestricted use, distribution, and reproduction in any medium, provided the original author and source are credited. 
as a reservoir for the spread of MRSA to patients and other HCWs [4]. Poor infection control measures are usually responsible in both acquiring and transmitting MRSA by HCWs.

This bacterium can establish solid interactions with nasal epithelial cells via various proteins and many cell surface components, thus transforming into persistent carriage [5]. The anterior nares are the main reservoir of MRSA, although other body sites are frequently colonized, such as the hands, skin, axillae, and intestinal tract [6].

The risk of MRSA transmission via transiently colonized hands of permanent nasal MRSA carriers in HCWs to the patients or hospital environment is three to six times greater than non-carriers and transient carriers [7].

Nasal colonization is considered crucial in the pathogenesis of MRSA infection acting as the reservoir for infection. S. aureus colonizes the anterior nares of $20 \%$ to $80 \%$ of the human population [8]. The rate of nasal carriage of S. aureus and MRSA varying from $16.8 \%$ to $90 \%$ among hospital personnel has been reported [9]. Healthcare workers who are asymptomatic nasal carriers can sometimes be the source of MRSA outbreaks [10].

Identification of patients and HCWs (in outbreak settings) colonized with MRSA, combined with hand hygiene and other precautions have been shown to be effective in reducing the transmission and controlling the spread of MRSA.

\section{Materials and Methods}

\section{Ethical approval}

Proposal of study was approved by the Institution board university of Azad Jammu and Kashmir Muzaffarabad and Ethical committee of Rawalpindi Institute Cardiology (RIC).

\section{Setting}

The study was carried out at Pathology department of a tertiary care hospital in Rawalpindi Pakistan.

\section{Study design}

It is a prospective as well as descriptive study.

\section{Study duration}

The study was carried over the period from 1st July to 1st November.

\section{Study subjects}

Inclusion criteria

Only coagulase positive S. aureus isolates were dealt in the study.

\section{Exclusion criteria}

All coagulase negative isolates were excluded from the study.

\section{Sample size}

Specimens were collected from $200 \mathrm{HCWs}$ working in hospital including Doctors and Medical officers (MO) of different wards, Anesthesia MOs, OT Perfusionists, OT Technicians and Nurses from different wards, OT dressers, ward boys and Ayas from different departments.

\section{Sample collection}

Sterile, moist cotton swabs were used for the collection of samples from anterior nares. The swab were taken from 1-2 cm inside of each nostril after rotating it 2-3 times both clockwise and anticlockwise with gentle pressure for 3-5 The swabs were cultured on blood agar (BA), Mannitol salt agar (MSA) and Macconkey agar (MA) \& incubate at $37_{\circ} \mathrm{C}$ for $24-48$ hours.

Repeat nasal \& throat swabs were taken from MRSA positive HCWs after one-week following treatment to ensure that he or she was no longer a carrier:

- Kept at non-patient contact areas or sent on leave to avoid transmission of infection.

- They were advised to bath twice daily using Triclosan for 5 days.

- $1 \%$ Chlorhexidine application on skin.

- Mupirocin $2 \%$ nasal ointment to be applied to each nostril, 3 times a day.

\section{Results}

\section{Sex wise distribution of MRSA nasal carriage}

Out of two hundred nasal specimens taken from HCWs, 48(24\%) were males and 152 (76\%) were females.

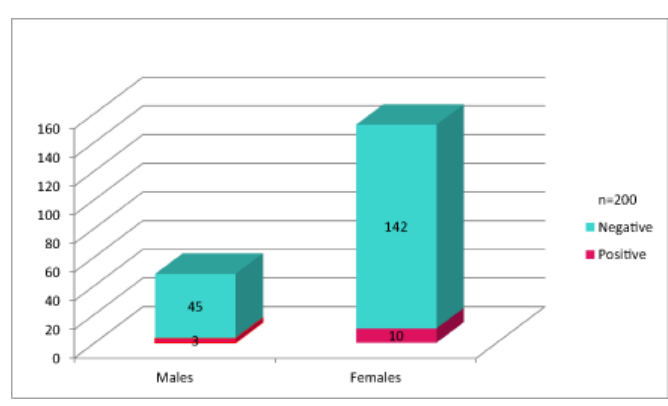

Figure 1: Sex wise distribution of MRSA nasal carriage.

\section{MRSA nasal carriage among HCWs in different departments}

Samples were collected from different departments of RIC. Specimens were taken from $91 \mathrm{HCWs}$ working in intensive care unit (ITC), 53 from operation theatre (OT), 33 from cardiac surgical ward (CSW), 12 from surgical private ward (SPW) and 11 from pediatrics department.

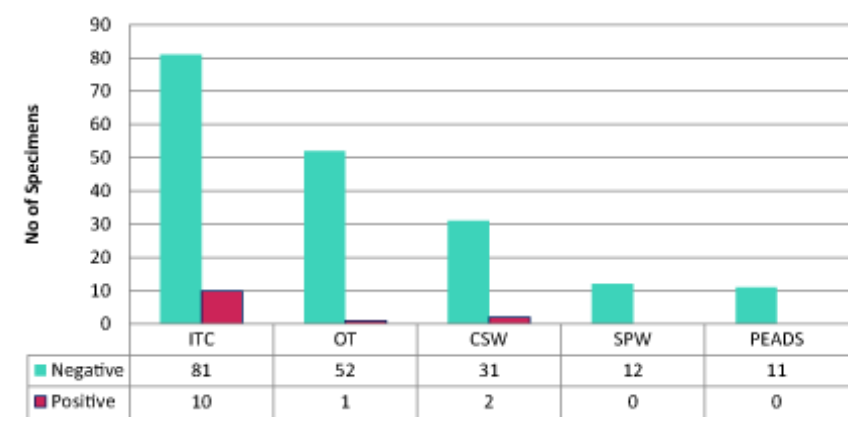

Figure 2: Nasal carriage of MRSA in HCWs of different departments.

The frequency of nasal carriage among HCWs in ITC, OT and CSW was $11 \%, 1.8 \%$ and $6.0 \%$ respectively, while there was no MRSA carrier in Surgical Private Ward and pediatrics department.

\section{MRSA Nasal carriage among different HCWs groups}

Specimens were collected from different HCWs i.e. doctors (11), anesthetists (13), Medical officers (MO) (11), OT perfusionists (9) and 
operation theatre technicians (OT) (12), nurses (120), dressers (3), ward boys (11) and ayas (10).

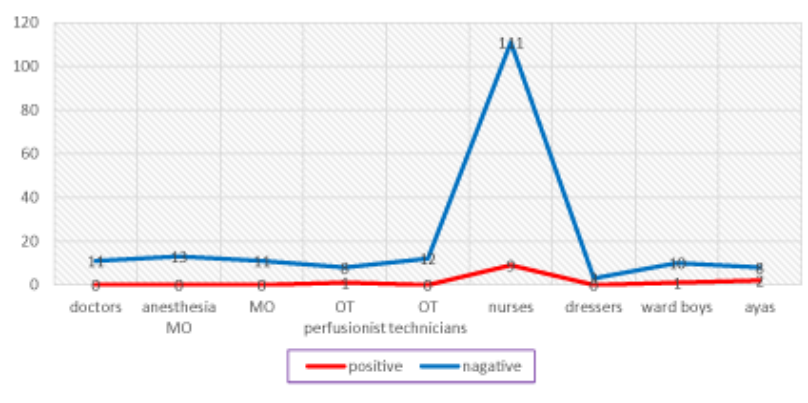

Figure 3: MRSA Nasal carriage among different HCWs.

The frequency of nasal carriage of MRSA in nurses was $7.5 \%, 9.09 \%$ in ward boys, $20 \%$ in ayas and $11.1 \%$ in perfusionists.

\section{Results of repeat samples}

All repeat samples of nasal $\&$ throat swabs collected after treatment were MRSA negative.

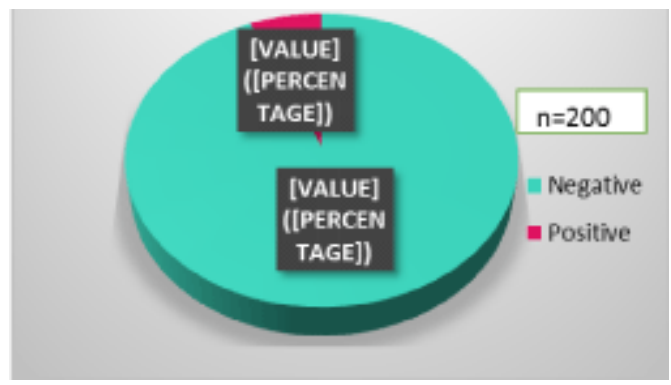

Figure 4: Frequency of MRSA nasal carriage before treatment.

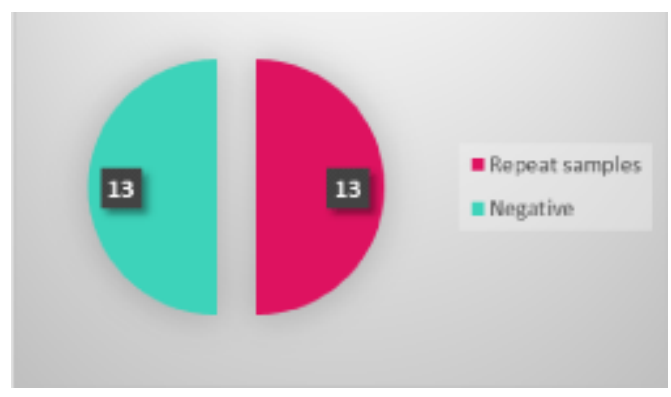

Figure 5: Results after treatment.

\section{Discussion}

Staphylococcus aureus (S. aureus) is one of the major human pathogens that can cause community and hospital-acquired (HA) infections. This bacterium is the most prevalent isolate taken from hospitalized patients. The global emergence of methicillin-resistant Staphylococcus aureus (MRSA) has turned into a serious public health problem as it is resistant to different antibiotics. Antibiotic therapy has faced severe difficulties due to these strains [11].

Nasal S. aureus have been implicated in community associated infections like soft tissue infections and hospital infections like bacteremia. It can colonize skin, armpits and particularly anterior nares. S. aureus including MRSA carriage are well known risk factor for subsequent infection. HCWs colonized with MRSA may carry these virulent hospital strains in their nose and skin and may transmit these organisms to the community creating a more dreadful situation [12].
With a few exceptions, the incidence of nosocomial infection caused by MRSA continues to increase worldwide.

The MRSA frequency reported from different countries of the worlds are given in table:

Table 1: Frequency of MRSA in different countries.

\begin{tabular}{ll}
\hline Countries & Frequency of MRSA \\
\hline United States of America & $59.0 \%$ \\
\hline Ireland & $54.7 \%$ \\
\hline India & $52.9 \%$ \\
\hline United Kingdom & $42.5 \%$ \\
\hline Iran & $37.2 \%$ \\
\hline China & $36.6 \%$ \\
\hline Sweden & $2.1 \%$ \\
\hline Netherlands & $1 \%$ \\
\hline
\end{tabular}

Table shows highly variable frequency of MRSA among different parts of the world [13].

The first case of MRSA in Pakistan was reported in 1989. Its prevalence has been reported to be from as high as $42 \%$ to as low as $7.5 \%$ in Pakistan [14].

It is necessary to detect MRSA carriers among health care workers (HCWs) in hospitals, particularly those who work in critical care areas. These individuals act as a potential source of infection to their patients, causing nosocomial infections and extended stays in hospital. The best methods which can be used for controlling this are regular screening of HCWs and taking the appropriate preventive measures [15].

Dynamics and prevalence of MRSA colonization are affected by several factors such as type of hospital department, MRSA prevalence among patients, and inadequate compliance with infection control measures; these differ depending on geographical locations [16]. The prevalence of MRSA carriage of HCWs was estimated as $4.6 \%$ in a study carried in the DutchGerman border region in non-outbreak situations [17]. Studies have shown a prevalence of MRSA carriage among different HCWs populations at $8.7 \%$, $7.5 \%$ and $6.8 \%$ in Peru, North India and Taiwan respectively [18].

The prevalence of nasal carriage of S. aureus among hospital personnel has varied between $28.2 \%$ and $44.5 \%$ in different studies conducted in Iran. Similar studies conducted by Goyal et al. (Delhi) and Alghaithy et al. (Abha, Saudi Arabia) reported 6.6\% and 18.3\% MRSA carriers, respectively [19].

HCWs of three major hospitals of Peshawar district and the general population were screened for MRSA colonization in a study carried out in Pakistan. The study observed a high prevalence in nasal mucosa both in HCWs and in the general population as compared to colonization in the armpit [20].

HCWs colonized with MRSA act as a main reservoir for transmission of MRSA to patients and other HCWs. Spread of MRSA to the community leads to more alarming condition.

In our study, out of two hundred nasal specimens, 13 (6.5\%) were positive for MRSA. The frequency of MRSA nasal carriage was $6.2 \%$ and $6.6 \%$ in males and females respectively. The highest MRSA nasal carriage was observed in ITC department (11\%) in nurses having a frequency of $80 \%$ and $20 \%$ in ayas/maids. MRSA nasal carriage in CSW was $6.0 \%$ with $50 \%$ 
nurses and $50 \%$ ward boys while in OT only 1 perfusionist had nasal carriage of MRSA. Our study shows highest percentage of MRSA among nurses working in ITC. However, among different professional groups it was highest in ayas (20\%) followed by perfusionists $11.1 \%$, ward boys $9.09 \%$ and nurses $7.5 \%$. The highest percentage in ayas and ward boys may be due to their contact with infected patient's hands, clothes and equipment. Poor infection control measures and unawareness of infection control practices and lack of hand hygiene lead to frequent acquisition and transmission of MRSA among these groups.

Studies to find out MRSA carriage among health care workers has been reported from different hospital settings in different parts of the world.

In UK, the prevalence of MRSA colonization among tested HCW in the CCU/ICU was $3.7 \%$ which is quite lower then percentage found at RIC [21].

Surveys carried out in Germany showed MRSA prevalence between 0.4 to $4.5 \%$ among healthcare staff in different medical institutions [22].

MRSA prevalence rates among HCWs in Europe and the United States showed $4.3 \%$ and $6.6 \%$ respectively. The MRSA rate was highest in nursing staff (6.9\%) [23].

Alghaity et al reported MRSA carriage of $18.3 \%$ in 1999 in Abha, Saudi Arabia. MRSA carriage was $4.3 \%$ in a tertiary care hospital in Southeastern China with the highest frequency reported in nurses $(8 / 107 ; 7.5 \%)$. MRSA Carriage rate among surgical HCWs was 4.3\% (9/210) [24]. The overall MRSA carriage among HCWs in a tertiary care hospital in India was $8.5 \%$ and it was highest among nurses $10.41 \%$ (5/48). Nasal carriage of MRSA among male and female HCW's were $5.55 \%(3 / 54)$ and $9.58 \%(14 / 146)$ respectively [25].

A study conducted in University hospital in Tehran, Iran reported MRSA prevalence of $22.5 \%$ [26]. Whereas in Al-Shifa hospital in Gaza strip 31\% of HCWs carried S. aureus, $25.5 \%$ of which were identified as MRSA carriers. The MRSA carriage among nurses was $30.4 \%$ whereas it was $16 \%$ among doctors [27]. However in our study there was no MRSA carrier among doctors which shows their good hygiene and well awareness to the infection control measures.

In a study conducted in Holy Family Hospital, the nasal carriage of MRSA among HCWs is $1.5 \%$ with highest carriage rate in surgical ICU (40\%). The carriage rate in staff nurses was reported as $22.7 \%$ [28].

Other studies also show similar findings such as highest prevalence of MRSA in nurses as well as in ICU and HCWs working in surgical units. However, the prevalence of MRSA among HCWs is lowest in Netherlands and Holy Family Hospital Pakistan. In UK, Germany, Europe, China MRSA prevalence ranges between 0.4-4.5percent. The prevalence of MRSA among HCWs in US and India is $6.6 \%$ and $8.5 \%$ respectively which is similar to that observed in our study but the highest frequencies have been reported in University of Tehran, Iran and Al-Shifa hospital Gaza strip.

Luckily no MRSA carrier was found in Surgical private and Pediatrics departments. Moreover, no MRSA nasal carrier was seen in any Doctors, Anesthesia Medical officers, OT technicians and dressers.

The procedures of screening and decolonization for colonized HCWs remain controversial [29]. Although in regions with low MRSA prevalence, such as the Netherlands, screening after each contact with MRSA-positive patients is recommended [30], the guidelines of several European countries and North American health associations are more reluctant and only advocate staff screening in selected situations, such as epidemiological outbreaks [31]. Decolonization of nasal colonized HCWs with mupirocin is recommended by most guidelines, but critical questions have arisen about the systematic use of this antibacterial agent [32].

Other issues related to the management of colonized HCWs have been raised in the literature, including the questions of the optimal timing of HCWs screening and whether and for how long colonized HCWs should be excluded from work [33]. Work restrictions for HCWs colonized with MRSA differ geographically, ranging from being allowed to work without restrictions other than compulsory hand hygiene, to being removed from clinical duties or being forced to take leave of absence. In case of persistent carriers, further employment of the employee is not advised when there is patient contact. HCWs with MRSA colonization might therefore suffer from job restrictions.

\section{Conclusion}

High percentage of nasal carriage of MRSA among HCWs in our ITC department and CSW reflects compromise in following infection control measures especially hand hygiene. There is need to educate HCWs by regular training on infection control practices. The compliance with the sanitary and antibacterial guidelines of the health professionals is the single most important factor in preventing such infections. Simple preventive measures like hand washing before and after the patient examination, the use of sterile aprons and masks in the postoperative wards, awareness during the examination of the immunocompromised patients, and avoiding touching one's nose during work, can reduce the disease transmission rate considerably.

\section{References}

1. de San, Nour, Denis, Olivier, Gasasira, Marie-Fabrice, and De Mendonca, et al. "Controlled evaluation of the IDIMRSA assay for detection of colonization by methicillin resistant Staphylococcus aureus in diverse mucocutaneous specimens". J Clin Microbio/45(2007): 1098-1101.

2. Schmidt, Aurélie, Bénard, Stève, and Cyr, Sonyal. "Hospital cost of staphylococcal infection after cardiothoracic or orthopedic operations in France: a retrospective database analysis". Surg Infect 16(2015): 428-435.

3. Kumar, P, Shukla, I, and Varshney, S. "Nasal screening of healthcare workers for nasal carriage of coagulase positive MRSA and prevalence of nasal colonization with Staphylococcus aureus". Biol Med3(2011): 182-186.

4. Kirecci, E, Ozer, A, Aral, M, and Miraloglu, M. "A Research of nasal methicillin resistant/sensitive Staphylococcus aureus and pharyngeal betahaemolytic Streptococcus carriage in midwifery students in Kahramanmaras, Eastern Mediterranean Region of Turkey". Ethiop J Health Dev 2(2010): 57-60.

5. Mulcahy, Michelle E, and McLoughlin, Rachel M. "Host-bacterial crosstalk determines Staphylococcus aureus nasal colonization". Trends Microbiol 24(2016): 872-886.

6. Acton, DS, Plat-Sinnige, MT, Van Wamel, W, and De Groot, N, et al. "Intestinal Carriage of Staphylococcus Aureus: How Does Its Frequency Compare With That of Nasal Carriage and What Is Its Clinical Impact?". Eur J Clin Microbiol Infect Dis 28(2009): 115.

7. Coia, JE, Duckworth, GJ, Edwards, DI, and Farrington, M, et al. "Guidelines for the control and prevention of meticillin-resistant Staphylococcus aureus (MRSA) in healthcare facilities". J Hosp Infect 63(2006): S1-S44.

8. Brown, AF, Leech, JM, Rogers, TR, and McLoughlin RM. "Staphylococcus aureus colonization: modulation of host immune response and impact on human vaccine design". Front Immuno/4(2014): 507.

9. Gomes, IM, Marlow, MA, Pinheiro, MG, and Freitas, Mde F, et al. "Risk factors for Staphylococcus aureus and methicillin resistant S. aureus colonization among health care workers in pediatrics departments". $A m \mathrm{~J}$ Infect Contro/42(2014): 918-920.

10. Lamanna, O, Bongiorno, D, Bertoncello, L, and Grandesso, S, et al. "Rapid containment of nosocomial transmission of a rare community-acquired methicillin-resistant Staphylococcus aureus (CA-MRSA) clone, responsible for the Staphylococcal Scalded Skin Syndrome (SSSS)". Ital J Pediatr 43(2017): 5.

11. Lari, AR, Pourmand, MR, Ohadian Moghadam, S, and Abdossamadi, Z, et al. "Prevalence of PVL-containing MRSA isolates among hospital staff nasal carriers". Lab Med 42(2011): 283-286. 
12. Adhikari, S. "Nasal Carriage Rate of Staphylococcus Aureus and Mrsa among Health Care Workers in Tertiary Care Center". (2017).

13. Asghar, M, Asghar, N, Mumtaz, S, and Khan, SA, et al. "Frequency of methicillin resistant staphylococcus aureus (mrsa) colonization amongst hospital staff in teaching hospitals of Peshawar". Res J Med Sci 24(2016): 194-198.

14. Rafiq, MS, Rafiq, MI, Khan, T, and Rafiq, M, et al. "Effectiveness of simple control measures on methicillin resistant Staphylococcus aureus infection status and characteristics with susceptibility patterns in a teaching hospital in Peshawar". J Pak Med Assoc 65(2015): 915-920.

15. Srivalli, B, Ekyshwarya, S, and Setty, CR. "Nasal carriage of Methicillin Resistant Staphylococcus aureus (MRSA) among health care workers in a tertiary care hospital". Int J Biol Med Res 9(2018): 6348-6351.

16. Pourramezan, N, Moghadam, SO, and Pourmand, MR. "Methicillin-resistant Staphylococcus aureus tracking spread among health-care workers and hospitalized patients in critical wards at a university hospital, Tehran, Iran". New microbes and new infections 27(2019): 29-35.

17. Buenaventura-Alcazaren, FA, dela Tonga, A, Ong-Lim, A, and Destura, RV. "Prevalence and molecular characteristics of MRSA nasal carriage among hospital care workers in a tertiary hospital in the Philippines". J Microbiol Immunol(2019)

18. Askarian, M, Zeinalzadeh, A, Japoni, A, and Alborzi, A, et al. "Prevalence of nasal carriage of methicillin-resistant Staphylococcus aureus and its antibiotic susceptibility pattern in healthcare workers at Namazi Hospital, Shiraz, Iran". Int J Infect Dis 13(2009): e241-e247.

19. Asghar, M, Asghar, N, Mumtaz, S, and Khan, SA, et al. "Frequency of methicillin resistant staphylococcus aureus (mrsa) colonization amongst hospital staff in teaching hospitals of Peshawar". Res J Med Sci 24(2016); 194-198.

20. Papastergiou, P, and Tsiouli, E. "Healthcare-associated transmission of Panton-Valentine leucocidin positive methicillin-resistant Staphylococcus aureus: the value of screening asymptomatic healthcare workers". BMC Infect Dis 18(2018); 484

21. Ruscher, C. "Empfehlungen zur Prävention und Kontrolle von Methicillinresistenten Staphylococcus aureus-Stämmen (MRSA) in medizinischen und pflegerischen Einrichtungen " . BundesgesundheitsblattGesundheitsforschung-Gesundheitsschutz 57(2014); 695-732.

22. Dulon, M, Peters, C, Schablon, A, and Nienhaus, A. "MRSA carriage among healthcare workers in non-outbreak settings in Europe and the United States: a systematic review". BMC Infect Dis 14(2014); 363.
23. Kong, Y, Ye, J, Zhou, W, and Jiang, Y, et al. "Prevalence of methicillinresistant Staphylococcus aureus colonisation among healthcare workers at a tertiary care hospital in southeastern China". J Glob Antimicrob Res 15(2018); 256-261.

24. Srivalli, B, Ekyshwarya, S, and Setty, CR. "Nasal carriage of Methicillin Resistant Staphylococcus aureus (MRSA) among health care workers in a tertiary care hospital". Int J Biol Med Res 9(2018); 6348-6351.

25. Pourramezan, N, Moghadam, SO, and Pourmand, MR. "Methicillin-resistant Staphylococcus aureus tracking spread among health-care workers and hospitalized patients in critical wards at a university hospital, Tehran, Iran". New microbes and new infections 27(2019); 29-35.

26. El Aila, NA, Al Laham, NA, and Ayesh, BM. "Nasal carriage of methicillin resistant Staphylococcus aureus among health care workers at Al Shifa hospital in Gaza Strip". BMC Infect Dis 17(2017); 28.

27. Akhtar, N. "Staphylococcal nasal carriage of health care workers". J Coll Physicians Surg Pak 20(2010); 439-443.

28. Hawkins, G, Stewart, S, Blatchford, O, and Reilly, J. "Should healthcare workers be screened routinely for meticillin-resistant Staphylococcus aureus? A review of the evidence". Journal of Hos Infect77(2011); 285-289.

29. Vandenbroucke-Grauls, CMJE. "Methicillin-resistant Staphylococcus aureus control in hospitals: The Dutch experience". Infect Control Hosp Epidemiol 17(1996); 512-513.

30. Muto, CA, Jernigan, JA, Ostrowsky, BE, and Richet, HM, et al. "SHEA guideline for preventing nosocomial transmission of multidrug-resistant strains of Staphylococcus aureus and enterococcus". Infect Control Hosp Epidemio/24(2003); 362-386.

31. Verhoeven, PO, Grattard, F, Carricajo, A, and Lucht, F, et al. "An algorithm based on one or two nasal samples is accurate to identify persistent nasal carriers of Staphylococcus aureus". Clin Microbiol Infect 18(2012); 551-557.

32. Hawkins, G, Stewart, S, Blatchford, O, and Reilly, J. "Should healthcare workers be screened routinely for meticillin-resistant Staphylococcus aureus? A review of the evidence". J Hos Infect 77(2011); 285-289.

33. Albrich, WC, and Harbarth, S. "Health-care workers: source, vector, or victim of MRSA?" The Lancet Infect Dis 8(2008); 289-301.

How to cite this article: Ali AM, Kiyani AM, Hussain AB. "Methicillin Resistant Staphylococcus aureus Nasal Carriage among Health Care Workers at Rawalpindi Institute of Cardiology, Rawalpindi, Pakistan". J Infect Dis Med 5 (2020) doi: 10.37421/jidm.2020.5.141 